\title{
Editorial
}

\section{Sensors and Applications in Agricultural and Environmental Monitoring}

\author{
Yuan Li $\mathbb{D}^{1}{ }^{1}$ Ajit Ghosh, ${ }^{2}$ Zhenxing Zhang $\mathbb{D}^{3,4}{ }^{3}$ Zhifeng Yu, ${ }^{5}$ and Jingwei Wang ${ }^{6}$ \\ ${ }^{1}$ Northwest Land and Resources Research Center, Shaanxi Normal University, Xi'an, China \\ ${ }^{2}$ Max Planck Institute for Plant Breeding, Köln, Germany \\ ${ }^{3}$ Key Laboratory of Vegetation Ecology, Ministry of Education, Northeast Normal University, Changchun, China \\ ${ }^{4}$ State Environmental Protection Key Laboratory of Wetland Ecology and Vegetation Restoration, School of Environment, \\ Northeast Normal University, Changchun, China \\ ${ }^{5}$ The Ohio State University, Columbus, USA \\ ${ }^{6}$ Shanxi University of Finance and Economics, Taiyuan, China
}

Correspondence should be addressed to Yuan Li; liy681@nenu.edu.cn

Received 28 July 2021; Accepted 28 July 2021; Published 13 August 2021

Copyright (C) 2021 Yuan Li et al. This is an open access article distributed under the Creative Commons Attribution License, which permits unrestricted use, distribution, and reproduction in any medium, provided the original work is properly cited.

Sensors and applications in agricultural and environmental monitoring have become more essential to (1) farming practices, (2) crop growth estimation, (3) environmental changes, (4) global and regional observations on the earth's environment, and (5) monitoring/environmental analysis. Among the 31 papers submitted to this special issue, 22 original research articles, which address the sensors and applications in agricultural and environmental monitoring, have been published. The published papers can be summarized as follows.

J. Qi et al. in their paper entitled "Variation in Morphological and Physiological Characteristics of Wild Elymus nutans Ecotypes from Different Altitudes in the Northeastern Tibetan Plateau" identify the alteration in morphological and physiological characteristics by measuring photosynthetic physiology, nutrient content, and growth associated with adaptation of plants to conditions at different altitudes 2450, 2950, 3100, and $3300 \mathrm{~m}$ above sea level on the plateau. The authors conclude that the increased thermal regime experienced by plants introduced from high altitude to low altitude may facilitate the increased growth of Elymus nutans subtypes.

J. Fu et al. in their paper entitled "Accurate Variable Control System for Boom Sprayer Based on Auxiliary Antidrift System" utilize the auxiliary antidrift system of wind- curtain type air flow and the variable spraying control system for adaptive fertilizing and online measuring of working conditions. Their results showed that benefitting from the auxiliary air flow, the average utility of pesticide is improved from $26.76 \%$ to $37.98 \%$.

L. Liu et al. in their paper entitled "A Novel Method for Broiler Abnormal Sound Detection Using WMFCC and HMM" develop a method for broiler abnormal sound detection. Results showed that the developed recognition algorithm, using wavelet transform, Mel frequency, cepstrum coefficients (WMFCCs), correlation distance, Fisher criterion (CDF), and hidden Markov model (HMM), provided an average accuracy, precision, recall, and $F 1$ of $93.8 \%, 94.4 \%$, $94.1 \%$, and $94.2 \%$, respectively, for broiler sound samples.

$\mathrm{X}$. Su et al. in their paper entitled "A Review of Underwater Localization Techniques, Algorithms, and Challenges" unveil the existing challenges in the underwater environment.

B. Zhao et al. in their paper entitled "Online Measuring and Size Sorting for Perillae Based on Machine Vision" develop an approach based on the machine vision (MV) technique for online measuring and size sorting. Experimental results demonstrate that the average time consumption for a captured image, the average measuring error, the variance of measuring error, and the overall sorting accuracy 
are $204.175 \mathrm{~ms}, 1.48 \mathrm{~mm}, 0.07 \mathrm{~mm}$, and $93 \%$, respectively, implying the feasibility and satisfied accuracy of the proposed approach.

In the paper entitled "Development of an IoT-Based Indoor Air Quality Monitoring Platform" authored by J. Jo et al., an IoT-based indoor air quality monitoring platform, consisting of an air quality sensing device called "SmartAir" and a web server, is demonstrated. An application was developed to help in monitoring the air quality. Thus, approved personnel can monitor the air quality at any time and from anywhere, via either the web server or the application.

M. Li et al. in their paper entitled "Response Mechanism of Cotton Growth to Water and Nutrients under Drip Irrigation with Plastic Mulch in Southern Xinjiang" studied the effects of water and nutrient control measures on cotton plant height, stem diameter, biomass, seed yield, and soil moisture under an irrigated plastic mulch production system. It is suggested that deficit irrigation at $60 \sim 80 \%$ of the potential evapotranspiration at the flowering stage and 16-5.6-2.4 $\left(\mathrm{N}-\mathrm{P}_{2} \mathrm{O}_{5}-\mathrm{K}_{2} \mathrm{O}\right)$ fertilizer can be applied as an optimal water and nutrient management strategy to maximize the seed cotton yield, irrigation water efficiency, and overall growth and development of cotton.

X. Feng and J. Li in their paper entitled "Evaluation of the Spatial Pattern of the Resolution-Enhanced Thermal Data for Urban Area" chose three typical methods from the limited number of thermal sharpening methods designed for the urban area and made a comparison between them, together with a newly proposed thermal sharpening method, superresolution-based thermal sharpener. It is found that all methods obtained lower accuracy for data in winter than for data in other seasons. Linear water features and areas along it are difficult to be detected correctly using most methods.

$\mathrm{R}$. Ma et al. in their paper entitled "Colloidal Influence Factor of Bioflocculant in Coagulation of Chromium and Nitrobenzene" investigate the flocculation capacity of activated sludge flocculants in chromium ion and nitrobenzene solutions. It is found that compared with activated carbon, the activated sludge flocculant showed a good flocculation capacity for nitrobenzene, but poor flocculation for chromium following the initial concentration increase. The flocculation of nitrobenzene or chromium increased at the initial stage and decreased gradually following the primary dosage of flocculant increase. The flocculation for nitrobenzene increased at the primary stage and decreased after the peak, while the flocculation for chromium increased following the $\mathrm{pH}$ increase of both solutions.

K. N. Qureshi et al. in their paper entitled "Optimized Cluster-Based Dynamic Energy-Aware Routing Protocol for Wireless Sensor Networks in Agriculture Precision" propose a Gateway Clustering Energy-Efficient Centroid-(GCEEC-) based routing protocol where a cluster head is selected from the centroid position and gateway nodes are selected from each cluster.

M. Xu and J. Zhou in their paper entitled "Elite Immune Ant Colony Optimization-Based Task Allocation for Maximizing Task Execution Efficiency in Agricultural Wireless Sensor Networks" propose an elite immune ant colony opti- mization (EIACO) to deal with the problem of task allocation optimization, which is motivated by immune theory and elite optimization theory. Simulation results show that the proposed elite immune ant colony optimization has a better task execution efficiency and higher convergence speed than genetic algorithm and simulated annealing.

M. Zhang et al. in their paper entitled "Effects on Local Temperature and Energy of Oasis City Expansion in Arid Area of Northwest China" study the climate effect of urban expansion located in oases in arid area of Northwest China by surface and $2 \mathrm{~m}$ urban heat island (UHI) intensity and available energy ratio. The results show that $2 \mathrm{~m}$ UHI always displays positive twin peaks during the whole day, while surface UHI only displays a positive single peak for several hours during daytime at four seasons in the four years.

N. Lu et al. in their paper entitled "Leymus chinensis Tolerates Mowing Disturbance by Maintaining Photosynthesis in Saline-Alkali Heterogeneous Habitats" tested the effects of mowing intensity on plant photosynthesis under different heterogeneous patches with different saline-alkali soils. The results imply that moderate grazing or mowing can be used to maintain the productivity and economic benefits of grassland when soil heterogeneous patches with moderate salinealkali conditions.

G. Gao et al. in their paper entitled "Stem Water Content for Crape Myrtle in Response to Drought, Cold, and Disease Stress" selected Crape myrtle as an experimental subject and its stresses on stem water content were observed in four experiments including no stress, drought, cold, and disease stress. The authors conclude that the stresses on stem water content can be used as a qualitative evaluation index of the degree of the three types of stress.

$\mathrm{H}$. Xiang et al. in their paper entitled "Sensors Applied for the Detection of Pesticides and Heavy Metals in Freshwaters" briefly introduce the pollution status of two major pollutants, i.e., pesticides and heavy metals, in freshwaters worldwide. The results imply that future development of pesticides and heavy metal sensors should (1) enhance the sensitivity to meet the requirements for the protection of aquatic ecosystems and human health and (2) cover more diverse pesticides and heavy metals especially those toxic pollutants that are widely used and frequently are detected in freshwaters (e.g., glyphosate, fungicides, zinc, chromium, and arsenic).

H. Liang et al. in their paper entitled "Variation Characteristics of Stem Water Content in Lagerstroemia indica and Its Response to Environmental Factors" studied the change characteristics of stem water content (StWC) in the plant and its response to environmental factors. The results showed that the StWC of Lagerstroemia indica varies regularly day and night during the growth cycle. Meanwhile, the rising time, valley time, and falling time of StWC were various at the different growth stages of Lagerstroemia indica.

$\mathrm{J}$. Li et al. in their paper entitled "Change Analysis of Spring Vegetation Green-Up Date in Qinba Mountains under the Support of Spatiotemporal Data Cube" propose a new data model based on data cube technologies to efficiently organize remote sensing phenology and related reanalysis data in different scales. This research provided a new approach for analyzing phenology phenomena and its 
changes in Qinba Mountains that had the same reference value for other regional phenology studies.

H. Malon et al. in their paper entitled "Application of Low-Cost Sensors for the Development of a Methodology to Design Front-End Loaders for Tractors" developed a methodology to design tractor front-end loaders with a view of obtaining their accurate design during the bucket loading process. The experimental results obtained by means of low-cost sensors fitted to the front-end loader allow analyzing the existing strains at the points measured as well as validating the numerical model developed.

F. Zeng et al. in their paper entitled "A Novel Bioflocculant from Raoultella planticola Enhances Removal of Copper Ions from Water" studied copper ions in the water of Songhua River flowing through the chemical and living areas of Jilin City. The results showed that copper ions in the water of Songhua River flowing through the chemical and living areas of Jilin City show that the copper concentration in this area is between $0.066 \mathrm{ppb}$ and $0.159 \mathrm{ppb}$. The copper content index of the three water samples did not exceed the Class III standard of the Surface Water Quality Standard.

$\mathrm{P}$. Su et al. in their paper entitled "Anonymous and Efficient Certificateless Multirecipient Signcryption Scheme for Ecological Data Sharing" propose a solution to the key escrow problem and aim to improve efficiency on the basis of ensuring security by adopting elliptic curve cryptography. A rigorous security analysis proves that the scheme can resist various security attacks and ensure privacy protection based on random oracle model. Performance analysis also shows that this scheme has the advantage of computational overhead compared to the same type of scheme.

T. Sharmilan et al. in their paper entitled "Electronic Nose Technologies in Monitoring Black Tea Manufacturing Process" introduce an electronic nose to find the optimum level of fermentation detecting the variation in aroma level. It is found that the systems developed are capable of detecting the variation of aroma level using an array of metal oxide semiconductor gas sensors using different statistical and neural network techniques successfully.

Y. Wu et al. in their paper entitled "A Model with Leaf Area Index and Trunk Diameter for LoRaWAN Radio Propagation in Eastern China Mixed Forest" developed a model for leaf area index and trunk diameter. The results show that the $433 \mathrm{MHz}$ LoRa path loss in the mingled forest could be precisely predicted by our proposed model.

\section{Conflicts of Interest}

The editors declare that they have no conflicts of interest regarding the publication of this special issue.

\section{Acknowledgments}

We wish to thank all the researchers who have contributed in their great work to the success of this special issue. Also, we would like to thank the reviewers that have been involved in the reviewing process. We would like to acknowledge the editorial board members for approving the publication of this special issue.

Yuan $\mathrm{Li}$

Ajit Ghosh

Zhenxing Zhang

Jingwei Wang

Zhifeng $\mathrm{Yu}$ 